\title{
STATISTICAL PROPERTIES OF THE GALEX-SDSS MATCHED SOURCE CATALOGS, AND CLASSIFICATION OF THE UV SOURCES
}

\author{
Luciana Bianchi, ${ }^{1}$ Lino Rodriguez-Merino, ${ }^{1}$ Maurice Viton, ${ }^{2}$ Michel Laget, ${ }^{2}$ Boryana Efremova, ${ }^{1} \mathrm{James}_{\text {Amerald }},{ }^{1}$ \\ Alberto Conti, ${ }^{3}$ Bernie Shiao, ${ }^{3}$ Armando Gil de Paz, ${ }^{4}$ Samir Salim, ${ }^{5}$ A. Thakar, ${ }^{1}$ Peter G. Friedman, ${ }^{6}$

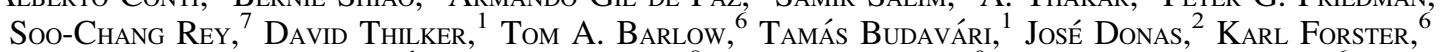 \\ Timothy M. Heckman, ${ }^{1}$ Young-Wook Lee, ${ }^{8}$ Barry F. Madore, ${ }^{9}$ D. Christopher Martin, ${ }^{6}$ \\ Bruno Milliard, ${ }^{2}$ Patrick Morrissey, ${ }^{6}$ Susan G. Neff, ${ }^{10}$ R. Michael Rich, ${ }^{5}$ \\ David Schiminovich, ${ }^{6}$ Mark Seibert, ${ }^{6}$ Todd Small, ${ }^{6}$ Alex S. Szalay, ${ }^{1}$ \\ Ted K. Wyder, ${ }^{6}$ Barry Y. Welsh, ${ }^{11}$ and Sukyoung K. YI ${ }^{8}$ \\ Received 2006 July 21; accepted 2006 October 26
}

\begin{abstract}
We use the Galaxy Evolution Explorer (GALEX) Medium and All-Sky Imaging Survey (MIS and AIS) data from the first public data release (GR1), matched to the Sloan Digital Sky Survey (SDSS) DR3 catalog, to perform source classification. The GALEX surveys provide photometry in far- and near-UV bands and the SDSS in five optical bands $(u, g, r, i, z)$. The GR1/DR3 overlapping areas are $363(86) \mathrm{deg}^{2}$ for the GALEXAIS (MIS), for sources within the $0.5^{\circ}$ central area of the $G A L E X$ fields. Our sample covers mostly $|b|>30^{\circ}$ Galactic latitudes. We present statistical properties of the GALEX-SDSS matched sources catalog, containing $>2 \times 10^{6}$ objects detected in at least one UV band. We classify the matched sources by comparing the seven-band photometry to model colors constructed for different classes of astrophysical objects. For sources with photometric errors $<0.3 \mathrm{mag}$, the corresponding typical AB-magnitude limits are $m_{\mathrm{FUV}} \sim 21.5, m_{\mathrm{NUV}} \sim 22.5$ for AIS, and $m_{\mathrm{FUV}} \sim 24, m_{\mathrm{NUV}} \sim 24.5$ for MIS. At AIS depth, the number of Galactic and extragalactic objects are comparable, but the latter predominate in the MIS. On the basis of our stellar models, we estimate the GALEX surveys detect hot white dwarfs throughout the Milky Way halo (down to a radius of $0.04 R_{\odot}$ at MIS depth), providing an unprecedented improvement in the Galactic WD census. Their observed surface density is consistent with Milky Way model predictions. We also select low-redshift QSO candidates, extending the known QSO samples to lower magnitudes, and providing $z \approx 1$ candidates for detailed $z \approx 1$ follow-up investigations. SDSS optical spectra available for a large subsample confirm the classification for the photometrically selected candidates with $97 \%$ purity for single hot stars, $\approx 45 \%$ (AIS) or $31 \%$ (MIS) for binaries containing a hot star and a cooler companion, and about $85 \%$ for QSOs.
\end{abstract}

Subject headings: Galaxy: stellar content — quasars: general — stars: statistics — surveys — ultraviolet: stars — white dwarfs

\section{INTRODUCTION}

Several recent and ongoing survey projects are providing a wealth of new data that allow us to refine theoretical models, and our global understanding of stars, galaxies, and the evolution of the universe. The Galaxy Evolution Explorer (GALEX; Martin et al. 2005) is performing the first survey of the sky in two broad bands: the far-ultraviolet (FUV) and the near-ultraviolet (NUV). The Sloan Digital Sky Survey (SDSS) is scanning one-fourth of

\footnotetext{
1 Department of Physics and Astronomy, The Johns Hopkins University, 3400 North Charles Street, Baltimore, MD 21218; bianchi@pha.jhu.edu.

${ }^{2}$ Laboratoire d'Astrophysique de Marseille, BP 8, Traverse du Siphon, 13376 Marseille Cedex 12, France.

3 Space Telescope Science Institute, Baltimore, MD 21218.

4 Universidad Complutense, Madrid, Spain.

5 Department of Physics and Astronomy, University of California, Los Angeles, CA 90095 .

${ }^{6}$ California Institute of Technology, MC 405-47, 1200 East California Boulevard, Pasadena, CA 91125.

7 Department of Astronomy and Space Science, Chungnam National University, Daejeon 305-764, Korea.

8 Center for Space Astrophysics, Yonsei University, Seoul 120-749, Korea.

9 Observatories of the Carnegie Institution of Washington, 813 Santa Barbara Street, Pasadena, CA 91101.

${ }^{10}$ Laboratory for Astronomy and Solar Physics, NASA Goddard Space Flight Center, Greenbelt, MD 20771.

11 Space Sciences Laboratory, University of California at Berkeley, 601 Campbell Hall, Berkeley, CA 94720.
}

the sky in five optical bands, $u, g, r, i$, and $z$. Bianchi et al. (2005a) explored the potential of the multiband photometric catalogs to classify astrophysical objects, using GALEX early release data, and showed that the UV bands are very sensitive to the detection of hot stellar objects and to the interstellar extinction by dust, as well as to the identification of low-redshift QSOs, as expected. Star counts are important because they delineate Galactic structure, and thus give clues to galaxy formation and evolution. The GALEX UV surveys provide unbiased detection of evolved, hot post-AGB objects, and of binary stellar systems containing white dwarfs (WDs), which will allow us to populate the observational Hertzprung-Russell diagram of hot evolved objects. Late evolutionary phases are extremely important for the yield of processed elements and the consequent chemical evolution of the interstellar medium (ISM). However, the post-AGB phases are much less understood than the early evolutionary phases, due to the scarcity of known objects. Such evolved objects are elusive in surveys at longer wavelengths both because of their low luminosity (at optical wavelengths) and high effective temperatures, to which optical colors are insensitive, and because they are extremely shortlived. We use the GALEX deep sensitivity to significantly extend the census of hot evolved objects in the Milky Way (MW).

In this paper we analyze the characteristics of a database we obtained by matching the GALEX GR1 (MIS and AIS) and SDSS DR3 (Abazajian et al. 2005) imaging catalogs. Both the 
GALEX GR1 and the SDSS DR3 data for the matched sources are available from the MAST archive. In $\S 2$ we describe the characteristics of the GALEX and SDSS photometric data used in this work. In $\S 3$ we describe our final catalog of GALEX-SDSS matched sources. In $\S 4$ we use color-color diagrams for classification of the sources. The results are discussed in $\S 5$.

\section{OBSERVATIONS}

This work makes use of photometric data collected by two of the largest astronomical surveys currently in progress, the GALEX mission and the SDSS project. Here we recall the basic characteristics of the imaging data from the two instruments, then we describe the matched source catalog constructed for this analysis.

\subsection{GALEX Data}

The GALEX mission (Martin et al. 2005) is performing a series of imaging and spectroscopic sky surveys in two ultraviolet bands, FUV and NUV. The instrument consists of a $50 \mathrm{~cm}$ diameter modified Ritchey-Chrétien telescope providing a very wide field of view $\left(1.25^{\circ}\right.$ diameter $)$ with good astrometric quality across most of the field, and a resolution of $\approx 4.2^{\prime \prime}-5.3^{\prime \prime}$ for FUV-NUV (Morrissey et al. 2007). In this work we limit the analysis to the sources in the inner $0.5^{\circ}$ radius of the GALEX field of view, for best astrometric and photometric quality.

The GALEX photometric data cover the wavelength range from 1344 to $2831 \AA$ with two broad bands, the FUV passband $(1344-1786 \AA)$ with $\lambda_{\text {eff }}=1528 \AA$ and the NUV band $(1771-$ $2831 \AA$ ) with $\lambda_{\text {eff }}=2271 \AA$ (Morrissey et al. 2005). Imaging surveys are carried out with different depth and coverage. In this work we use data from the All-Sky Imaging Survey (AIS), that has typically $\sim 100 \mathrm{~s}$ exposures with a $5 \sigma$ NUV limiting magnitude of $m_{\mathrm{NUV}} \sim 20.8$, and from the Medium Imaging Survey (MIS), with typical $\sim 1500 \mathrm{~s}$ exposures and limiting magnitude of $m_{\mathrm{NUV}} \sim 22.7$ in the AB system (Morrissey et al. 2005). The photometric system used in this paper is based on the AB magnitude scale (Oke \& Gunn 1983), although in the color-color diagrams we also provide the Vega-magnitude scale, to facilitate comparison with other work.

We note that the GALEX flux calibration has been revised since the early release data (used, e.g., by Bianchi et al. [2005a] in similar work), and the current processing and calibration brings a total shift in the FUV - NUV color of $\sim 0.12$ mag, such that the GR1 photometry is giving redder colors than the early release data by about this amount. Zero points of 18.82 and 20.08 mag for the FUV and NUV magnitudes, respectively (AB system), are used in the GALEX GR1 catalog.

\subsection{Sloan Digital Sky Survey}

The SDSS project (York et al. 2000) is mapping one-fourth of the sky in five broad optical bands: $u, g, r, i$, and $z$ (Fukugita et al. 1996), using a dedicated $2.5 \mathrm{~m}$ telescope with a wide-field of view and a $0.5 \mathrm{~m}$ telescope for photometric calibration. An automated image-processing system measures photometric and astrometric properties of each source (Pier et al. 2003).

The SDSS photometric system covers from 3000 to $11000 \AA$ with five nonoverlapping pass bands. The $u$ filter peaks at $3500 \AA$ with a width of $600 \AA$, the $g$ is a blue-green band centered at $4800 \AA$ with a width of $1400 \AA, r$ is a red band centered at $6250 \AA$ with a width of $1400 \AA, i$ is a far red filter centered at $7700 \AA$ with a width of $1500 \AA$, and the near-infrared passband $z$ is centered at $9100 \AA$ with a width of $1200 \AA$.
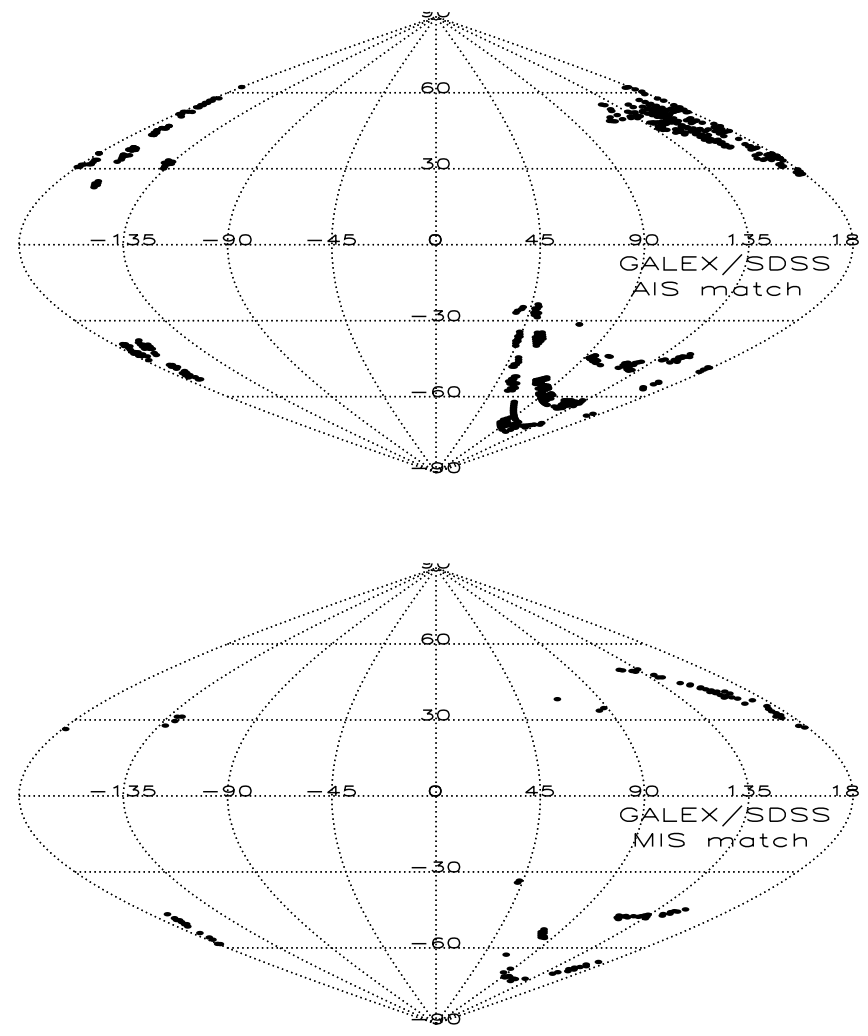

FIG. 1.-Locations of the common areas between the GALEX GR1 release and the SDSS DR3 release are shown in Galactic coordinates. Top: The All-Sky Imaging Survey carried out by GALEX has 622 fields matching the SDSS, the total unique area is $363 \pm 3 \mathrm{deg}^{2}$, when we restrict the GALEX fields to the central $1^{\circ}$ diameter. Bottom: The GALEX Medium Imaging Survey has 112 fields in common with SDSS DR3 covering a unique overlap area of $86 \pm 1 \mathrm{deg}^{2}$.

\section{THE GALEX-SDSS MATCHED SOURCE CATALOG}

In the GALEX GR1 release there are 622 AIS fields and 112 MIS fields that overlap areas of the sky observed by the SDSS. Figure 1 displays the GALEX-SDSS overlapping fields in Galactic coordinates. As a starting point we used a GALEX GR1SDSS DR3 matched catalog available from the MAST database. The match between GALEX and SDSS sources was done based on position using a $4^{\prime \prime}$ match radius (Budavari et al. 2004).

\subsection{Calculation of Areas of Overlap}

In order to calculate the density of sources from our classification work, we determined the total areas of overlap between the GALEX GR1 and SDSS DR3 releases with the following method. We use in our analysis only sources within the central $1^{\circ}$ of the GALEX field, therefore for each GALEX field we considered an effective radius of $0.5^{\circ}$. Partial overlap among some $G A L E X$ fields is not removed in the online GALEX database, hence sources observed in more than one field have multiple entries in the catalog. We wrote a code that scans the entire sky and calculates the unique area covered by the GALEX fields included in our matched catalog, and then calculates the part of this area covered also by the SDSS. Three subsequent steps are performed: (1) the celestial sphere is divided in small $\left(0.05^{\circ}\right.$ on a side), approximately square, elements, (2) the distance between the center of each area element in the grid to the GALEX field centers is used to determine whether the element was included in our survey, and thus contributed to the total area, and (3) a check is performed on each area element included in our GALEX coverage to assess whether it was also included in the area observed 
by the SDSS. The resulting unique-coverage areas of the matched catalogs are $363 \mathrm{deg}^{2}$ for AIS and $86 \mathrm{deg}^{2}$ for MIS. These figures will be used to determine the density of sources (number $\mathrm{deg}^{-2}$ ) in the next sections. Note that the total areas refer to a $0.5^{\circ}$ radius GALEX field, corresponding to our sample restriction. See also Figure 1 of Bianchi et al. (2006).

The error in our area determination was estimated by running several tests on a small area of the sky, including thousands of field position simulations. The maximum estimated uncertainty in the areas from our procedure is 3 and $1 \mathrm{deg}^{2}$ for AIS and MIS, respectively.

We note that the SDSS database sky partition (hierarchical triangular mesh $[\mathrm{HTM}]$ ) could not be used to estimate the area coverage due to the HTM's intrinsic inability to return accurate areas, and to an error in the areas and vertices of the triangular tassels, which prevented calculation of exact areas of the HTM tassels.

\subsection{The Final Catalog}

We used as a starting point the matched catalogs available from the MAST archive, which include all sources from the GALEX GR1 MIS and AIS, and from the SDSS DR3 releases, matched according to their position using a match radius of $4^{\prime \prime}$. The matched database contains a total of over 2 million objects (1.4 million from AIS and 0.9 million from MIS) detected in at least one UV band. In order to proceed to the analysis of the sources, we first eliminated multiple entries for the same source.

GALEX sources observed in more than one pointing result in multiple entries for the same source in the online catalog. Eliminating GALEX duplicate entries for the same source reduced the number of sources by $23 \%$ for AIS and by $15 \%$ for MIS. In addition, some GALEX sources have more than one SDSS counterpart due to the different spatial resolution: GALEX has a point-spread function (PSF) of $\sim 4.5^{\prime \prime}-6^{\prime \prime}$ while the SDSS PSF is $\sim 1.4^{\prime \prime}$. For these sources the analysis of photometric colors would be meaningless, since the GALEX photometry would be a composite measurement of different objects. Therefore, GALEX sources with multiple matches must be excluded from the classification analysis, but their fraction must be counted for source statistics. Removal of GALEX sources with multiple optical counterparts within the match radius reduced the number of sources by $13 \%$ for AIS and by $11 \%$ for MIS. Our object density counts ( $(5)$ take into account the fraction of sources eliminated because of having more than one optical counterpart. After eliminating duplicate GALEX detections, and UV sources with multiple SDSS matches, our final catalog contains 1,074,460 AIS sources and 752,190 MIS sources.

Finally, we must evaluate the possible contamination by spurious matches, and whether the purity of the sample would be improved by decreasing the match radius. In this work we considered all sources with a match radius up to $4^{\prime \prime}$ (but excluded multiple matches, as explained above).

When we apply error cuts of $0.15,0.1$, and $0.1 \mathrm{mag}$ (for FUV, NUV, and optical, respectively) to the sample, the distance between GALEX coordinates and SDSS coordinates is less than 2.5" for $99 \%$ (96\%) of the AIS (MIS) sources. If no error cuts are applied, a higher number of matched sources with GALEX and SDSS coordinates differing by more than $2.5^{\prime \prime}$ is found over the entire catalog. As a result of the photometric error cuts applied in our analysis, possible spurious matches are not expected to significantly affect the results.

In fact, the UV sky is rather "empty," that is, much more scarcely populated than the sky at optical wavelengths, due to the paucity of hot stars relative to the vast majority of cooler objects.
Therefore, a possible spurious match (positional coincidence) would probably occur if multiple optical sources are detected around the position of the UV source. Such cases are eliminated as explained above. Because we use both FUV and NUV bands, and our optical photometry includes the $u$ and $g$ bands, we expect that a hot object appearing as both an FUV and NUV source would be detected in $u$ and $g$. Random positional associations would be much more likely to occur if we considered matches of UV sources with red or IR bands. We did not impose in our final analysis samples that good photometric measurements exist for the UV sources also in $i$ and $z$ bands. Such requirement would considerably limit the final sample of hot stellar objects, in addition to possibly favoring spurious matches. A purity of over $95 \%$ for matches of UV sources with optical sources was predicted by simulations conducted during the design phases.

We also checked for possible artifacts in the matched catalog. Instrumental artifacts would likely be eliminated by the matching process itself, however a caveat remains about possible contamination of our samples by SDSS sources that the pipeline generates by "shredding" galaxies, that is, deblending extended objects into a number of individual photometric sources. We have investigated the problem, and we believe such sources cannot be reliably eliminated with the use of the current SDSS or GALEX photometric flags. Therefore we specifically searched whether any of our selected sources $(\S 5)$ falls within the radius of any known galaxy, using a list of galaxies with $R_{25}>1^{\prime}$ extracted from the catalog of de Vaucouleurs et al. (1991). We found only one case in our AIS catalogs of WDs, and three cases in the MIS catalog, of pointlike sources that are in fact portions of large galaxies. A few sources out of several thousand QSO candidates were also identified as "shredded galaxies."

\subsection{Properties of the Matched GALEX-SDSS Source Catalog: Analysis Sample Definition}

Figures 2-4 display the number of objects in our matched catalog as a function of magnitude for each band. The histograms show the total number of objects included in the final catalog (all detections), as well the numbers obtained by applying different error cuts. These histograms are useful for estimating the magnitude limits reached in restricted samples (by applying error cuts in the analysis), which are helpful for interpreting the results. In the histograms, we also marked the typical limiting magnitude for each band as given by the projects via the MAST and SDSS Web sites. Note that the SDSS pipeline generates sources several magnitudes below the nominal limits for each band, and it is surprising that the error cuts are not sufficient to eliminate these spurious detections. Figures 3 and 4 show that while the histograms of sources brighter than the magnitude limit behave as expected, with fainter sources progressively eliminated by more stringent error cuts, there is an almost specular distribution of spurious sources at the faint magnitudes, well below the expected SDSS limit. Such spurious sources are different in each band, therefore while they are not eliminated by error cuts in an individual band, they are eliminated by applying both error and magnitude cuts to the sample.

In the following section, we discuss the effects of error cuts applied as appropriate to separate the sources into classes of astrophysical objects based on their colors, in order to minimize contamination by other types of objects. Error cuts translate (statistically) into magnitude limits, in each photometric band, as can be inferred from the histograms in Figures 2-4. The number of objects remaining in our catalog when different error limits are applied in each band are given in Table 1. It is extremely important to consider the effects of the magnitude limits when using 

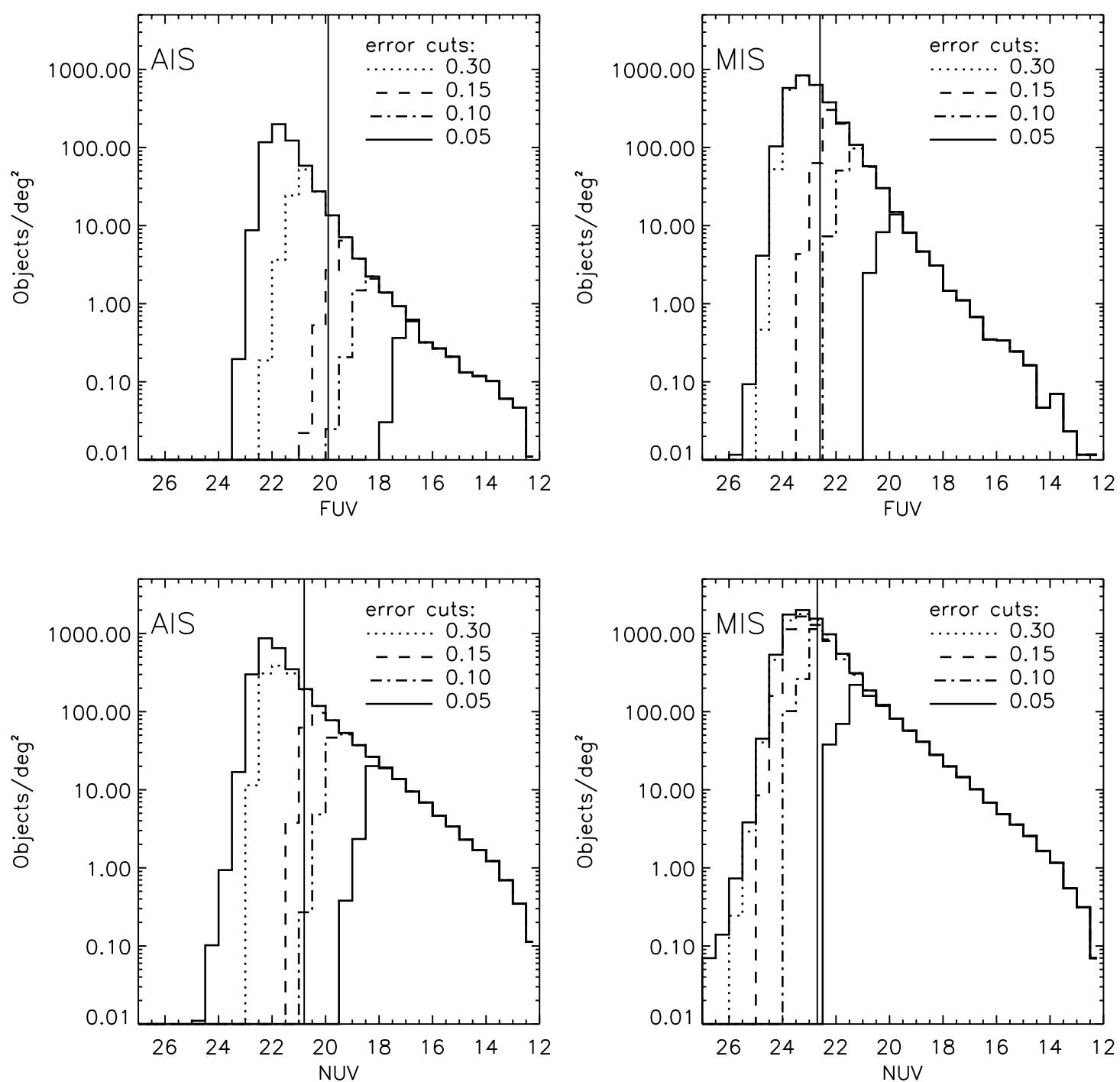

FIG. 2.-Distribution of sources from our GALEX-SDSS matched catalog as a function of UV magnitudes (top: FUV; bottom: NUV), separately for AIS and MIS. The solid line is the total number of sources (after duplicates have been removed as explained in the text), other lines show the histograms when error cuts are applied. Vertical lines display the $5 \sigma$ detection limit for a typical exposure (from the GALEX GR1 documentation via the MAST Web site).

colors to classify objects and to derive luminosity functions. Therefore, we provide some illustration of such effects and discuss them in $\S 4$.

In our analysis we also use SDSS spatial information to separate the sources into pointlike (at the SDSS $\sim 1.4^{\prime \prime} \mathrm{PSF}$ ) and extended sources. Pointlike sources are mostly stellar objects or QSOs (see $\S 4$ ), while extended sources are typically galaxies. The numbers of pointlike and extended sources are approximately equal at the depth of the AIS survey, but the number of extended sources (galaxies) increases significantly at fainter magnitudes (MIS survey) as shown in Figures 5-7. Likewise, among the pointlike sources, the low-redshift QSO candidates increase more than the number of hot star candidates at fainter magnitudes (§ 4.2).

\section{ANALYSIS: CLASSIFICATION OF THE GALEX SOURCES}

In this section we analyze the colors of the matched sources to extract information about their nature. Bianchi et al. (2005a) showed two sample color-color diagrams demonstrating that the combination of the GALEX UV bands with optical photometry is a powerful tool for identifying low-redshift QSOs, hot stellar objects, and binary stellar objects containing a hot WD.

\subsection{The Model Colors}

In Figures 5-7 we compare observed colors of our sources to model colors, calculated by applying the transmission curves of the GALEX and SDSS filters to theoretical spectra or templates of different astrophysical objects.

Theoretical stellar colors for main sequence and supergiant stars are derived from the stellar libraries of Lejeune (1997), L. Bianchi et al. (2008, in preparation), and Rodriguez-Merino et al. (2005), for a total of six metallicity values (two cases shown in the color-color diagrams: solar and one-tenth solar). All these grids are based on Kurucz (LTE, line-blanketed, plane-parallel) models. We compared, for sample cases, the broadband stellar colors derived from the Kurucz model grids, to colors computed from TLUSTY (Hubeny \& Lanz 1995) model atmospheres for hot stars (non-LTE, line-blanketed, plane-parallel), as well as with stellar atmospheres plus wind models (non-LTE, line-blanketed, spherical, hydrodynamic), computed by us (Bianchi \& Garcia 2002; Garcia \& Bianchi 2004; L. Bianchi et al. 2008, in preparation) 

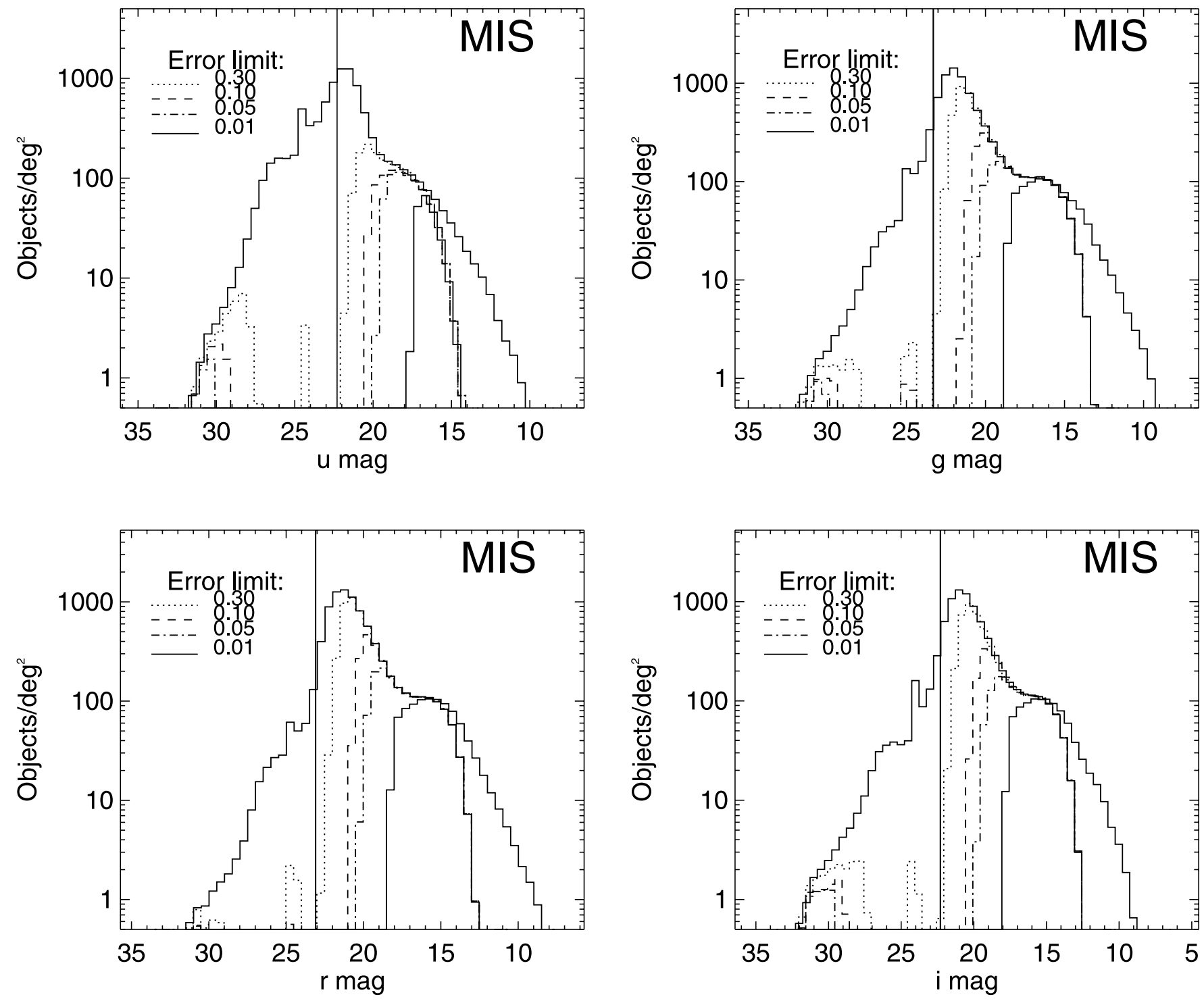

FIG. 3.- Similar to Fig. 2 for the SDSS filters. Only the sources matched to the GALEX MIS sources are shown, since the depth of the SDSS DR3 is obviously the

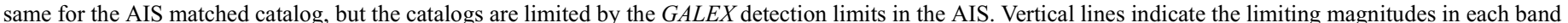
from the SDSS Web site. The solid line is the total number of objects (including objects with saturation). The other lines show the histograms when error cuts are applied.

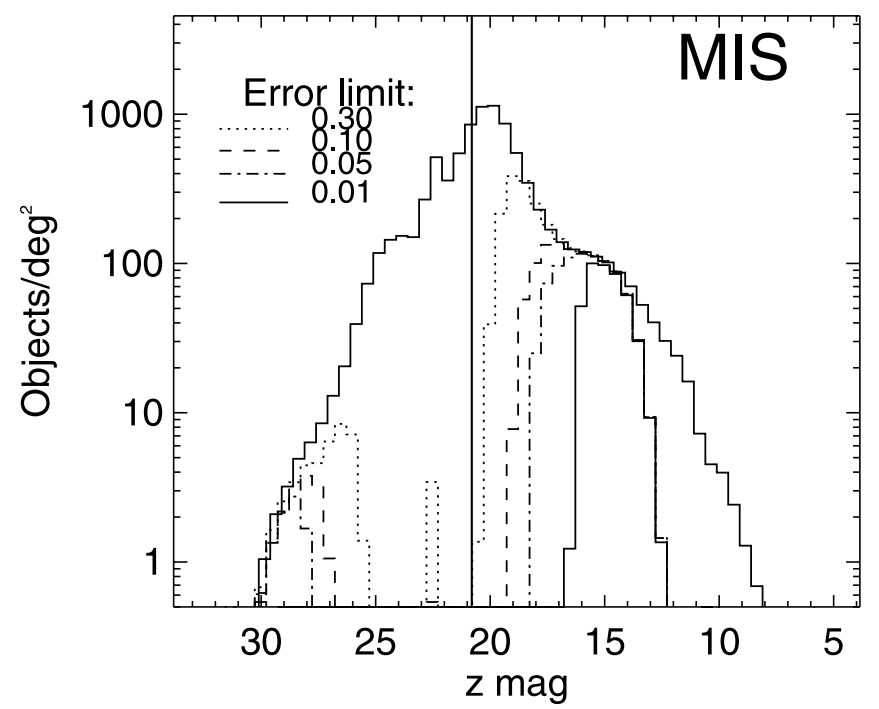

Fig. 4.-Similar to Fig. 3 for SDSS $z$ magnitudes. with the WM-basic code of Pauldrach et al. (2001). As expected, there is no appreciable difference in the broadband colors. The whole grid of models will be published elsewhere (L. Bianchi et al. 2008, in preparation). We calculated white dwarf (WD) models using the TLUSTY code of Hubeny \& Lanz (1995), for various gravities and solar, pure $\mathrm{H}$, and pure He abundances. In the color-color diagrams (Figs. 5-7), we show stellar model colors as a function of $T_{\text {eff }}$ for three different gravities (representing main-sequence [MS], supergiants [SG], and high-gravity stars with $\log g=9$ ), and two metallicity values, to illustrate the effects of relevant parameters without crowding the figure.

To define the locus of galaxies, we computed broadband colors from "simple stellar population" (SSP) models from the library by A. Bressan (2005, private communication), as well as from a library of galaxy templates. The templates were calculated for ellipticals, spirals, and irregulars (marked E, S, and I in the figures) using the GRAZIL code (Bressan et al. 1998; Silva et al. 1998). We show in Figures 5-7 these model colors as a function of age, for redshift $z=0$. For QSO colors, we used a composite model of Francis et al. (1991) revised as described in Bianchi et al. (2005a), as well as the SDSS average template, with 
TABLE 1

Statistics of Sources in the Different Bands

\begin{tabular}{|c|c|c|c|c|c|c|}
\hline \multirow[b]{3}{*}{ BAND } & \multicolumn{6}{|c|}{ Number of Sources } \\
\hline & \multicolumn{2}{|c|}{ (ERROR < $0.2 \mathrm{mag})$} & \multicolumn{2}{|c|}{ (ERROR < $0.1 \mathrm{mag})$} & \multicolumn{2}{|c|}{$($ ERROR $<0.05 \mathrm{mag})$} \\
\hline & AIS & MIS & AIS & MIS & AIS & MIS \\
\hline FUV .................... & 15,007 & 137,940 & 2902 & 23,840 & 821 & 3866 \\
\hline NUV ................... & 250,063 & 627,353 & 83,540 & 314,498 & 31,275 & 75,680 \\
\hline 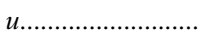 & 269,070 & 99,563 & 206,481 & 74,777 & 169,439 & 58,671 \\
\hline$g \ldots \ldots \ldots \ldots \ldots \ldots \ldots \ldots$ & 631,119 & 287,428 & 448,334 & 172,208 & 312,059 & 116,371 \\
\hline 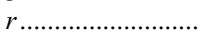 & 69,9744 & 355,341 & 513,223 & 211,791 & 350,779 & 132,569 \\
\hline$i \ldots \ldots \ldots \ldots \ldots \ldots \ldots \ldots$ & 637,288 & 310,262 & 448,761 & 182,945 & 309,971 & 117,935 \\
\hline$z$ & 365,375 & 144,429 & 255,060 & 96,299 & 194,863 & 73,006 \\
\hline
\end{tabular}
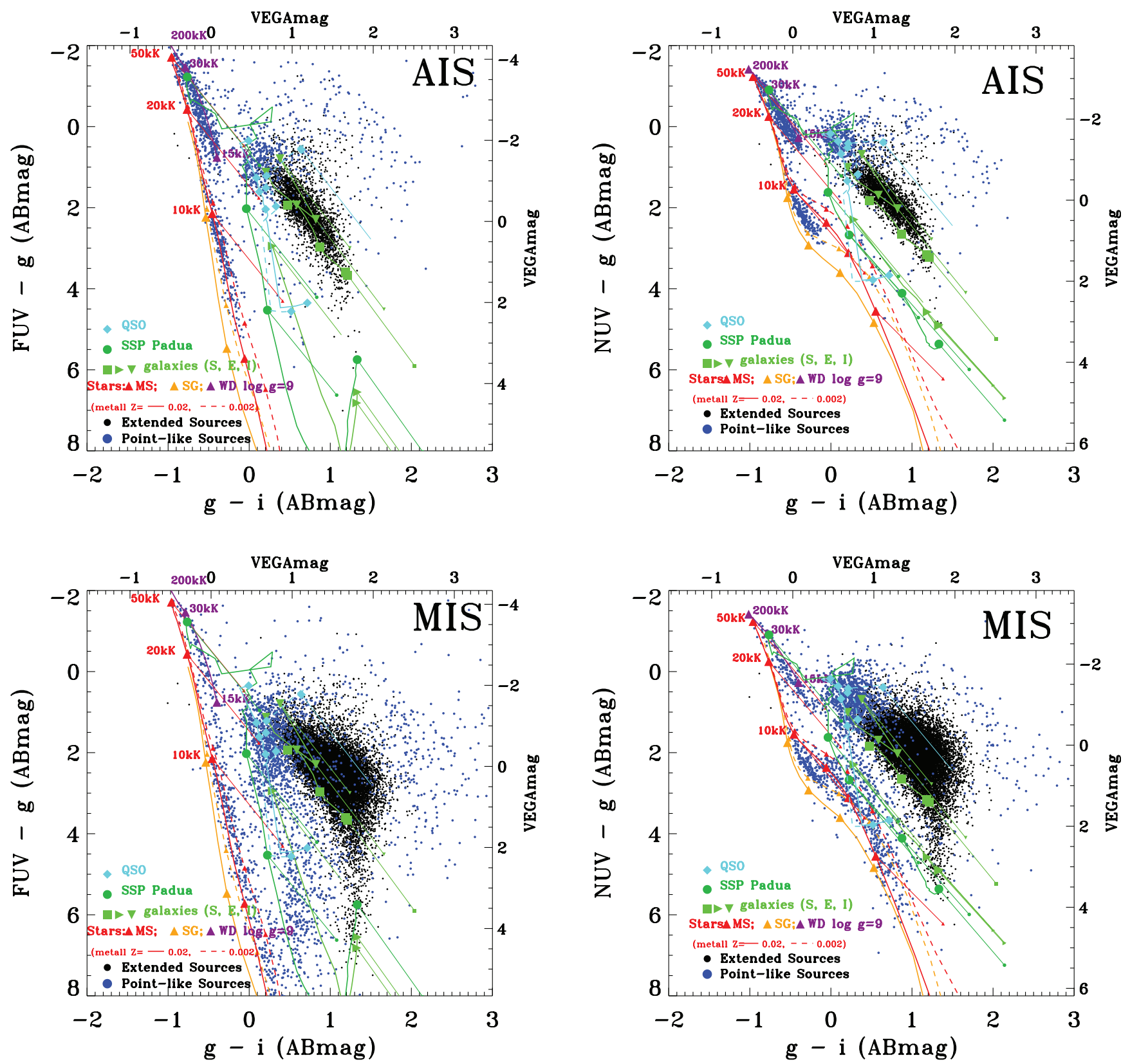

FIG. 5.-FUV $-g$ vs. $g-i$ and NUV $-g$ vs. $g-i$ color-color diagrams for the AIS and MIS sources. Blue dots are pointlike sources and black dots are extended

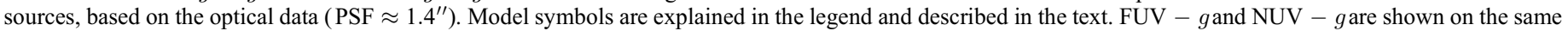

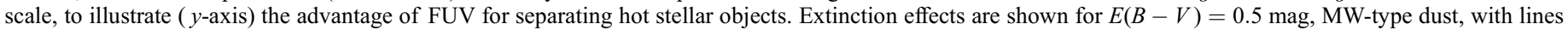

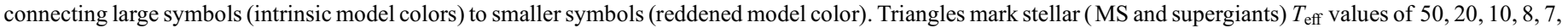

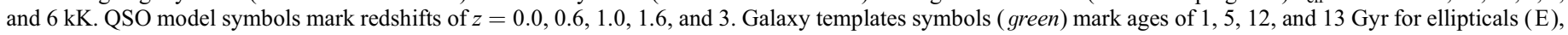
spirals (S), and irregulars (I). SSP marked ages are $1 \mathrm{Myr}, 200 \mathrm{Myr}, 500 \mathrm{Myr}, 1.5 \mathrm{Gyr}$, and 15 Gyr. Pointlike sources cluster along the stellar sequence (predominantly

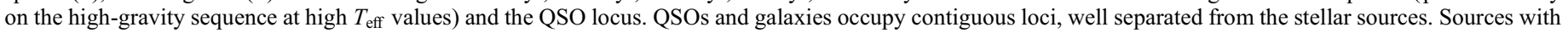
photometric errors better than $0.15 / 0.10 / 0.05 / 0.05 \mathrm{mag}$ in $\mathrm{FUV} / \mathrm{NUV} / g / i$ are shown. 

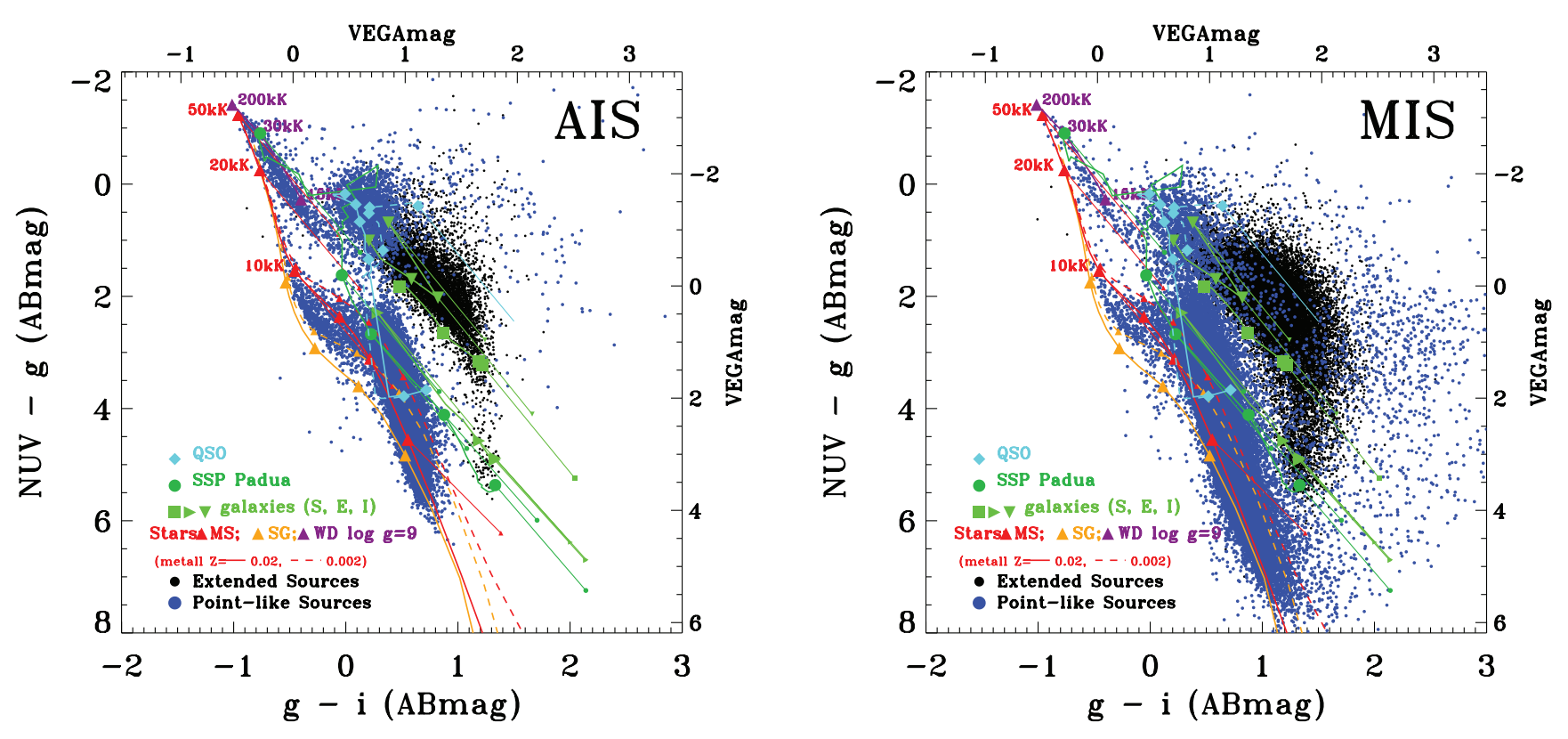

FIG. 6.-NUV $-g$ vs. $g-i$ color-color diagrams for the AIS and MIS sources, with symbols as in the previous figure. In this figure sources with errors $<0.1$ mag in NUV and $<0.05$ mag in the optical bands are shown, regardless of FUV-band photometric error. The comparison with the previous figure, where an error cut in the FUV band was also applied, illustrates the selection effects driven by FUV magnitude limits and error cuts in the analysis. While the hot objects hardly change, the lower $T_{\text {eff }}$ stellar sequence becomes extremely populated in these panels.

extrapolations at long and short wavelengths by W. Zheng (2006, private communication), that include Ly $\alpha$ absorption. Model colors from both these QSO templates are shown as a function of redshift in the color-color diagrams, the first plotted with a solid line, the latter with a dashed line.

Bianchi et al. (2005a) showed two sample color combinations, for the GALEX early data release sample, and also plotted a comparison of (known) spectroscopically classified objects with the model colors, showing that the loci defined by our models match very well the observed properties of each astrophysical class represented. We omit the comparison of the models to known objects in this work, as it would show the same result. Instead, in this paper we describe the effects of physical parameters, such as $T_{\text {eff }}$, metallicity, and gravity for stars. Below we discuss general properties and then we comment on individual color-color diagrams.

Effects of interstellar extinction by dust are applied to the model spectra, for varying amounts and types of extinction, in the calculation of the grids of model colors. Because we are using broadband photometry, applying extinction corrections $A_{\lambda} / E(B-V)$ for the $\lambda_{\text {eff }}$ of the filter bandpass to the intrinsic model magnitudes would only be an approximation of the reddening effects on the colors. Instead, we reddened each model spectrum with progressive amounts of $E(B-V)$, for different extinction laws, and then calculated broadband colors for the reddened model spectra. For the analysis of hot Galactic objects, and for the sample in this paper in particular, which includes fields at high Galactic latitudes, the extinction is small as we see in the next section. Therefore, we only show the case of MW-type extinction with $R_{V}=3.1$ in the color-color diagrams.

\subsection{Color-Color Diagrams}

Figures 5-7 show a number of color-color diagrams of the sources and model colors, chosen to illustrate the most relevant effects of the physical parameters and useful classification criteria, as well as the statistical properties of the sources.

In these figures, pointlike sources are shown with blue dots and extended sources with black dots. To avoid excessive crowd- ing in the diagrams, in general only sources with errors better than $0.15 \mathrm{mag}(0.10 \mathrm{mag})$ in FUV (NUV) and better than $0.05 \mathrm{mag}$ in the optical bands are shown. More details are given in the captions. Model colors are shown with different symbols and colors, explained in the figures. The effects of reddening by interstellar dust are shown with lines connecting the intrinsic model color (large symbols) to the reddened model color (smaller symbols), for a sample case of Galactic typical extinction with $R_{V}=3.1$, and for $E(B-V)=0.5 \mathrm{mag}$. It can be seen from all diagrams that for most hot stars the probable reddening does not exceed $E(B-V) \approx 0.1 \mathrm{mag}$, as can be expected given the high Galactic latitudes of the fields included in our catalog (Fig. 1).

\subsubsection{General Properties}

In all the color-color diagrams, but especially in the FUV NUV versus $g-r$ diagram (Fig. 7) and FUV $-g$ versus $g-i$ (or NUV $-g$ vs. $g-i$ ) diagram (Figs. 5 and 6 ), the extended sources (black dots; mostly galaxies) are well separated from the stellar sources (blue dots; pointlike sources, near the stellar model colors). The locus of the QSOs lies somewhat in between and is occupied in these diagrams by a compact cloud of mostly pointlike sources, populating the locus of the low- $z$ QSO template colors. A number of extended sources is also found in the same color space, which is also shared by the young-age SSP and elliptical galaxy models. Note that if we consider points with good photometry also in the FUV band, only the locus of low-z QSOs is populated with sources. Instead, sources with good photometry in the NUV-optical range (FUV drop-outs) do extend over the color-space of higher redshifts QSOs, as expected, and of stars with lower temperatures.

Another general feature evident in all diagrams, from the density of points in regions defined by the model colors for different objects, is that the number of extragalactic sources (galaxies and QSOs) increases much more than the number of Milky Way stellar sources at fainter magnitudes. This effect is quantified by the surface density plots shown in $\S 5$. 

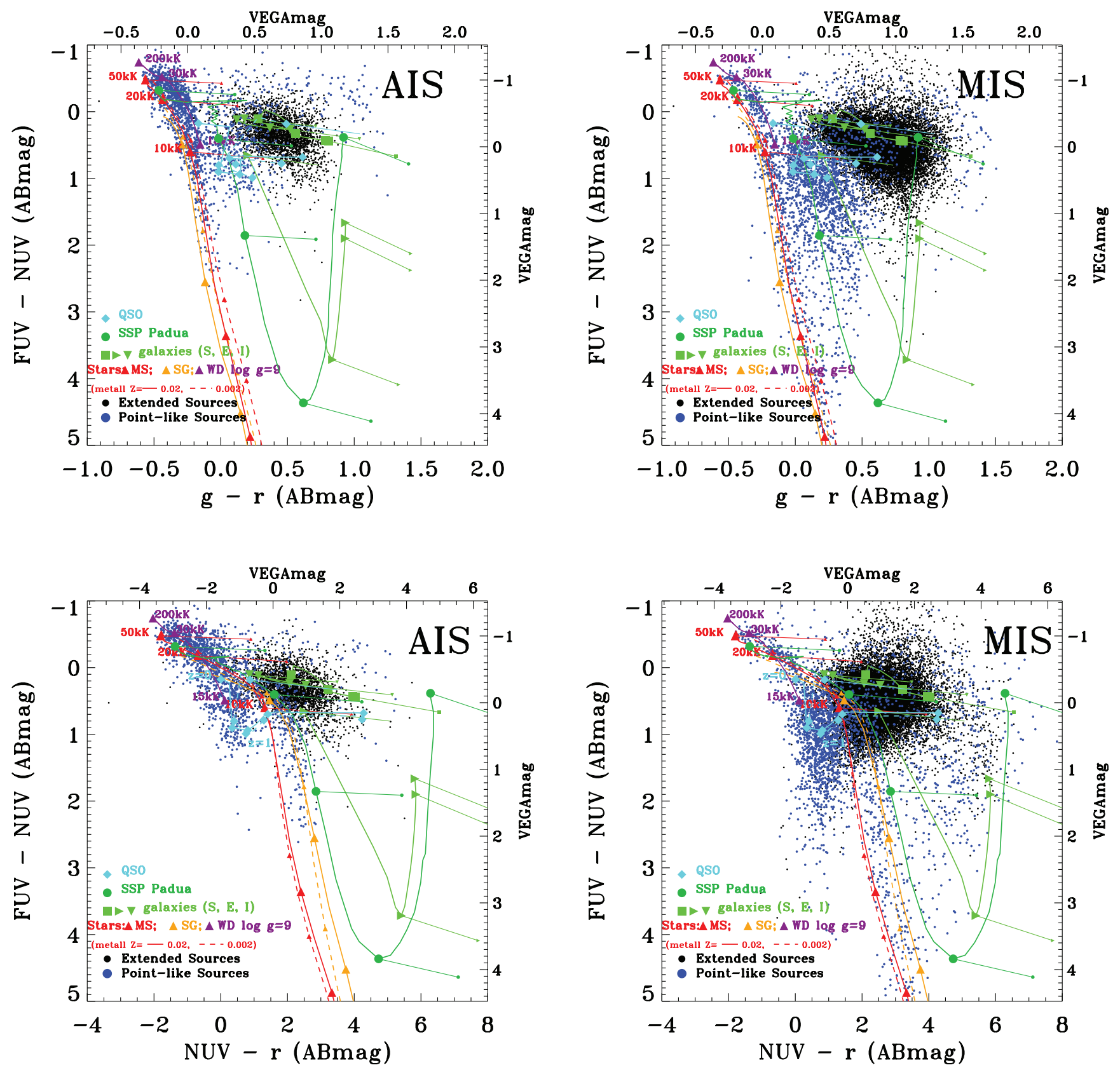

Fig. 7.-FUV - NUV vs. $g-r$ and FUV - NUV vs. NUV $-r$ diagrams. Symbols and colors as in previous figure. Stars and galaxies are well separated. The low- $z$ QSOs are in between stars and galaxies in the top diagrams but are more separated from both these classes of objects in the FUV - NUV vs. NUV $-r$ diagram, especially for redshifts around 1 (the lowest point in the "dip" of the QSO model colors). Here, QSOs at $z=0$ overlap with cool WDs, and QSOs with $z \approx 1.6$ have similar colors to A-type MS stars, but the selection of QSOs around $z=1$ is best performed by using this color combination. Cataclysmic variables at some stages (binaries containing a WD and an accretion disk) may contaminate the $z=1$ QSO locus. These objects are rare and can be recognized only spectroscopically. The FUV - NUV vs. NUV - $r$ diagram can also be used to separate single and binary hot WDs whose FUV - NUV colors are bluer than the QSOs.

The galaxies (extended sources; black dots) are well represented by the three "average" templates for irregulars, spirals, and ellipticals. Two properties of the sample can be inferred from the color-color diagrams. First, the templates cover well the space for irregular and spiral galaxies in the AIS sample, which is limited to brighter magnitudes. For the deeper MIS sample the templates' intrinsic colors rather provide an envelope to distribution of the sources, and the extinction effects shown (foreground MWtype) do not account for the entire extent of the data points on the color-color diagrams. This may be an indication of intrinsic mixed, possibly non-MW-type extinction. The analysis of galaxies colors from the GALEX surveys is not the scope of this work and is pursued in other works, therefore for our purpose it is sufficient that the unreddened galaxy colors produce an envelope to the observed distribution of the extended sources. The second point of relevance is that data points are seen with colors corresponding to ellipticals of either young or old ages, due to the contribution of hot $\mathrm{He}$ burning low-mass stellar population such as horizontal-branch stars and their progeny (Yi 2003) in the diagrams including the FUV band, in which these objects are probably too faint at intermediate ages. This is especially evident in the shallower AIS sample. When the more sensitive NUV band is considered (regardless of FUV detection of the source), the locus of ellipticals, especially at old ages, becomes more populated, even in the AIS survey (Fig. 6).

As for the stellar sources, our models for different luminosity classes (main sequence vs. supergiants) and metallicities show 

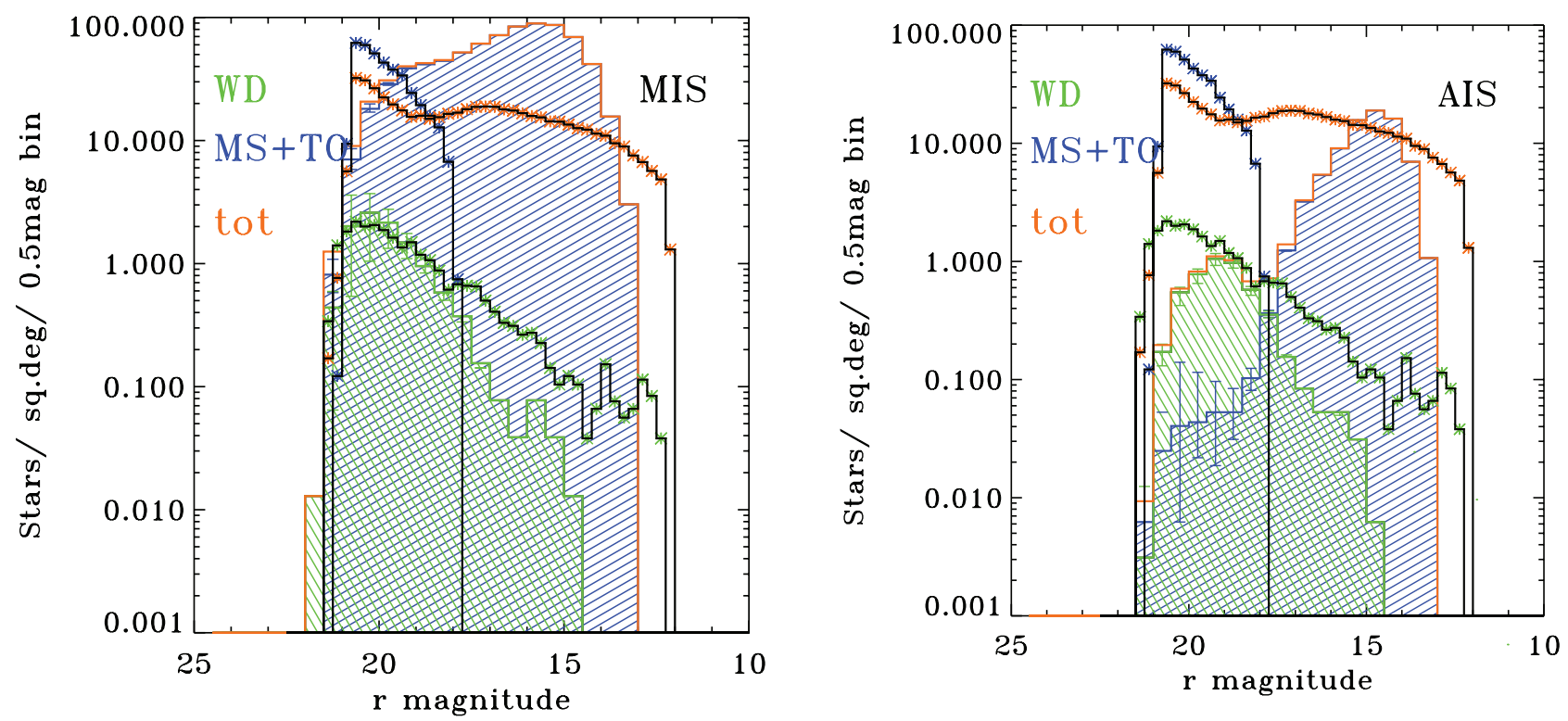

Fig. 8. - Surface density of WDs ( green) and lower gravity stars (blue). In orange, the sum of the two samples. The stars were selected from the NUV $-g$ vs. $g-r$ colors, for direct comparison with the Milky Way model (see text). We restricted the sample to sources with photometric errors better than 0.1 mag in NUV, $g$, and $r$, to avoid contamination by QSOs (see Fig. 5). The diagram on the left shows the sources extracted from the deeper MIS survey, the one to the right shows the sources from the AIS. The black histograms show the predictions from the Milky Way model (see text for details), with colored asterisks to indicate WD, non-WD, and total, respectively. Sources around 14th mag in optical bands may be affected by saturation in the SDSS survey and therefore excluded, so our histograms are incomplete for objects brighter than $r \approx 15 \mathrm{mag}$.

the separation of the photometric colors for stars cooler than approximately $10,000 \mathrm{~K}$, most evident in the NUV $-g$ versus $g-i$ diagram. For hotter stars, supergiants and main-sequence colors are indistinguishable; however, the well-defined cluster of stellar sources separate from the main-sequence colors is well represented by high-gravity stellar models. Only WD models with $\log g=9$ are shown for clarity. We see that the majority of the hottest stellar sources lies on the high-gravity sequence (evolved stars) rather than on the main-sequence or supergiant color sequence. An interesting point is that, for the case of MWtype extinction (as shown), reddening would displace the intrinsic color of a very hot star roughly along color sequence of high-gravity stars in the NUV $-g$ versus $g-i$ diagram, making $T_{\text {eff }}$ and reddening effects indistinguishable. However, the effects of $T_{\text {eff }}$ and reddening on the stellar colors are different in the FUV - NUV versus $g-r$ and FUV $-g$ versus $g-i$ diagrams and they can be disentangled. In any case, it is evident from all diagrams that the number of objects along the high-gravity stellar sequence increases at lower $T_{\text {eff }}$ values, consistent with numerical expectations. It would be unlikely that the majority of objects were extremely hot, highly reddened main-sequence or supergiant stars.

Another interesting stellar population component that can be easily detected, especially in the FUV - NUV versus NUV $-r$ diagram (Fig. 7), is that of stellar binaries composed of a white dwarf and a much cooler object. A significant number of pointlike sources can be seen with very blue FUV - NUV colors, but red optical colors. These objects appear as an arclike distribution of blue points on the top right part of the color-color diagrams, and are presumably WDs plus M-dwarf pairs. The WD+MD binary sequence has been first recognized at optical colors (e.g., Raymond et al. 2003; Smolcic et al. 2004; Pourbaix et al. 2004), but the UV bands are much more sensitive to the detection of systems with hot WDs. Smolcic et al. (2004) found $\approx 0.4$ of such binaries per square degree from the SDSS DR1. This population is discussed again in $\S 5.1$.
The last general point worth discussing, and often overlooked, is the effect of the photometry's magnitude limits (and hence also effects of error cuts) on the sample. Figures 5 and 6 show the same NUV $-g$ versus $g-i$ diagram, in one case selecting sources with good photometry in the four filters shown, regardless of detection in the FUV (Fig. 6). In Figure 5 the same sample is restricted to sources that also have good photometry (error better than $0.15 \mathrm{mag}$ ) in the FUV band. As expected, the hot star and low-redshift QSO samples do not change significantly; however, QSOs at higher redshift and a huge number of cooler stellar sources appear only when no FUV cut is applied. Interestingly, also a number of extended sources occupy the crowded locus of the lower $T_{\text {eff }}$ stellar sequence and higher $z$ QSOs. These data points are not visible in the diagram because the pointlike sources (the primary subject of investigation in this work) are plotted over the extended sources. In general, imposing good photometry also in the FUV band reduces the sample by a factor of about 10 , with respect to a sample based on NUV-band good photometry (see also Table 1). Obviously, magnitude limits affect objects differently depending on their colors.

\subsubsection{Discussion of the Individual Color-Color Diagrams}

We finally comment here on each diagram separately. The NUV $-g$ versus $g-i$ and FUV $-g$ versus $g-i$ color-color diagrams (Figs. 5 and 6) are somewhat similar to the classical optical $U-B$ versus $B-V$ diagram (e.g., Bianchi et al. 2005b) but present several advantages thanks to the broader wavelength range. Stars are well separated from galaxies and low-redshift QSOs in the $g-i$ color. Binary stars containing a hot WD and a much cooler companion are easy to detect (top right blue points in the diagram). The price to pay for the wide color separation of the objects is that the very hot WDs are faint in the $i$ band; thus, a sample selected from these colors is clean but limited to objects brighter than the detection depth that can be reached by the GALEX surveys (see next section). We plot the FUV $-g$ and $\mathrm{NUV}-g$ colors (left and right panels, respectively) on the same 

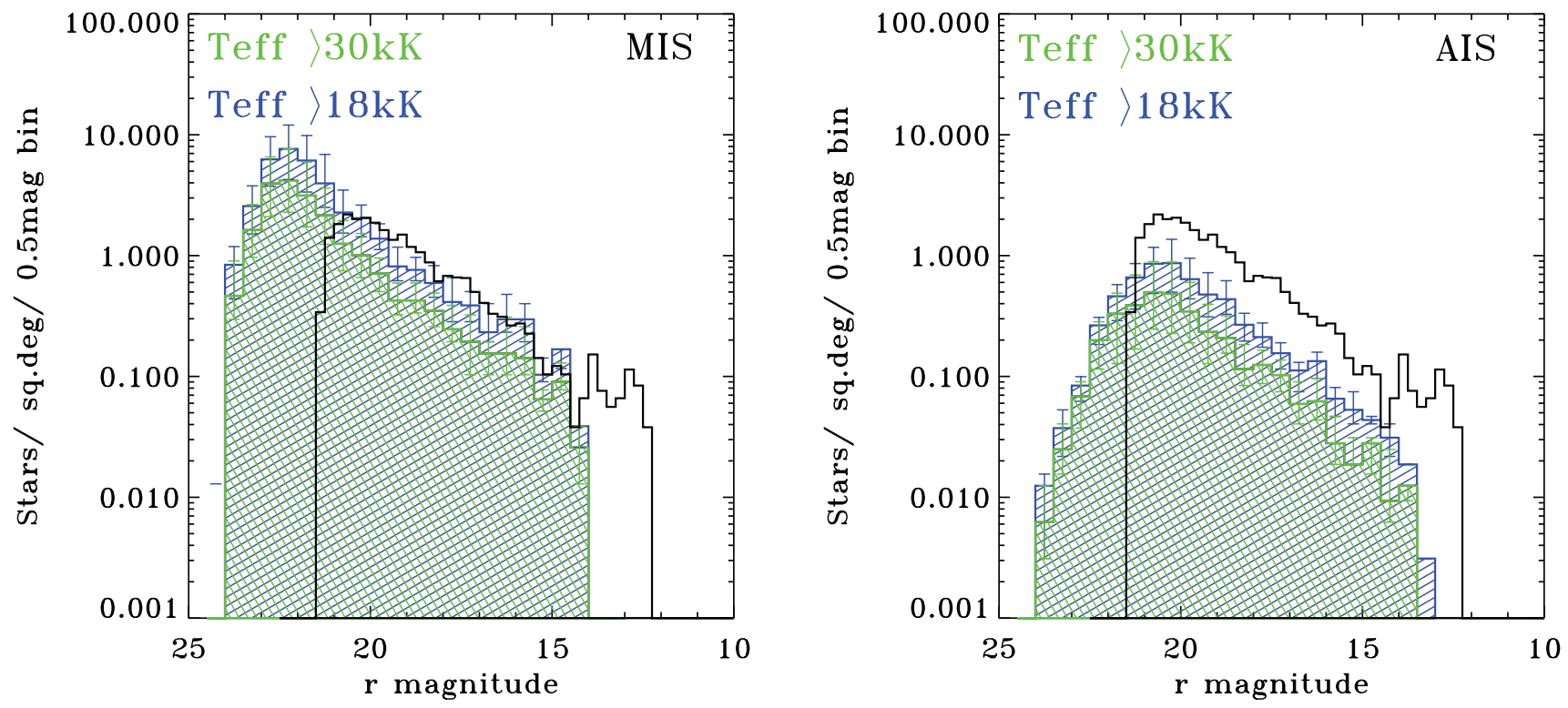

FIG. 9.- Surface density (in the $r$ band) of hot stars selected from the FUV - NUV (GALEX) color. The FUV - NUV color cuts correspond to $T_{\text {eff }}>18,000 \mathrm{~K}$ (blue histograms) and $T_{\text {eff }}>30,000 \mathrm{~K}$ ( green histograms) for stars with $\log g=5.0$, but include lower $T_{\text {eff }}$ values for stars of higher gravities (see text). Sources with photometric errors of $<0.3 \mathrm{mag}$ in FUV and NUV are included. This selection does not use SDSS measurements, thus avoiding the loss of bright stars due to saturation seen in the previous figure. A fraction of these objects have optical colors discrepant from the hot-WD UV colors, and they are either binaries with a cool companion or extragalactic objects intruded in the sample. The apparent difference in the AIS and MIS counts at bright magnitudes is due to these objects having an $r$ magnitude that does not correspond to that of a single WD (which is not taken into account by the Milky Way model), as proven by the next figure. For the same reason, the apparent match of the MIS number of objects with the model is probably biased in the $r$ band.

scale, to illustrate the advantage of including a FUV band as diagnostic for the hottest objects: note the much larger spread in color among hot temperature models, when the FUV band is included. Broadband photometric colors separate for stars of different gravity and metallicity only at $T_{\text {eff }}$ lower than $\approx 10,000 \mathrm{~K}$, as shown also by Bianchi \& Efremova (2006) in a recent $H S T$ study of stellar populations. For high-gravity stars, we plot only one sequence of model colors with $\log g=9$ for clarity. While supergiants and MS stars are indistinguishable at high $T_{\text {eff }}$ values, gravities much higher than $\log g=5$ (our model sequence plotted in red) are clearly separated photometrically. Most of the GALEX hot stellar sources cluster around the $\log g=9$ sequence and are therefore likely to be subdwarfs or WDs. A dramatic difference in the sources' census is made by including or neglecting the FUV photometry (Fig. 5 vs. Fig. 6); this effect has already been mentioned above. Finally, note the direction of the reddening effect on the colors in the NUV $-g$ versus $g-i$ diagram: it is very similar to the effect of the major physical parameters $\left(T_{\text {eff }}\right.$ for stars, age for galaxies, redshift for QSOs - in some ranges), making these particular colors unsuitable to disentangling reddening and, e.g., $T_{\text {eff }}$ for WDs. The degeneracy is less severe for the FUV $-g$ versus $g-i$ diagram, and it is completely removed in the diagrams including a FUV - NUV color (Fig. 7). Such extinction effects apply only to the case of MW-type dust, and would be slightly different for other types of dust.

Similar considerations apply to the FUV - NUV versus $g-r$ diagrams (Fig. 7, top). However, stars with different gravities are less separated, while galaxies, QSO and stars are better separated, with respect to the diagrams discussed previously.

The NUV $-r$ versus FUV - NUV diagram (Fig. 7, bottom) separates very well stellar objects from galaxies, thanks to the broad wavelength baseline. The stellar sequence is continuously populated, with the number of stellar objects fading out at $T_{\text {eff }}$ about $8500 \mathrm{~K}$ in the AIS catalog, but extending to cooler $\left(T_{\text {eff }} \sim\right.$ $6000 \mathrm{~K})$ stars in the deeper MIS catalog. Note that these limits are driven by imposing an error cut of $\mathrm{FUV}_{\text {err }}<0.15$ mag (as used in the color-color diagrams). In particular, hot stellar objects can be easily selected from their FUV - NUV color, while a UV $-r$ color allows us to separate single hot stars from binaries with a hot and a cool component. Using the NUV $-r$ and FUV - NUV colors, we extract hot star candidates in different $T_{\text {eff }}$ ranges, and low-redshift $(z<1.6)$ QSO candidates (next section). The distribution of hot stars and QSO candidates are shown in Figures 8-11.

In the next section we present the statistical properties of the hot stellar objects (single and binary) candidates. An analysis of the physical parameters of these objects will be the subject of future papers with spectroscopic follow-up.

\section{DISCUSSION AND CONCLUSIONS}

As discussed in the previous section, the color-color diagrams can be used to separate objects by astrophysical class, and in particular to select hot stars and low-redshift QSO candidates. In this section we derive surface densities for such objects and compare them to previous known catalogs, as well as to predictions by a Milky Way model.

\subsection{Hot Stars Candidates}

According to the fluxes of our TLUSTY models, a WD with $T_{\text {eff }}=50,000 \mathrm{~K}(100,000 \mathrm{~K}), \log g=7.0$, and $R=0.2 R_{\odot}$ has an $\mathrm{AB}$ magnitude (in absence of reddening) of $r=8.5,13.5$, 18.5 , and $20.0(7.9,12.6,17.9,19.4) \mathrm{mag}$ at $100 \mathrm{pc}, 1 \mathrm{kpc}, 10 \mathrm{kpc}$, and $20 \mathrm{kpc}$, respectively. Again from our models, its colors are $\mathrm{FUV}-r=-2.35(-2.62)$ and $\mathrm{NUV}-r=-1.75(-1.93$; AB mag) for $T_{\text {eff }}=50,000$ and $100,000 \mathrm{~K}$, respectively. A radius of $R=0.2 R_{\odot}$ would be a typical value for a very hot post$\mathrm{AGB} \operatorname{star}\left(T_{\mathrm{eff}} \approx 100,000 \mathrm{~K}\right)$ at the end of the constant-luminosity phase. A lower mass, cooler remnant may have a larger radius. A hot WD then would dim by several magnitudes descending (at approximately the same $T_{\text {eff }}$, initially) along the cooling sequence, 

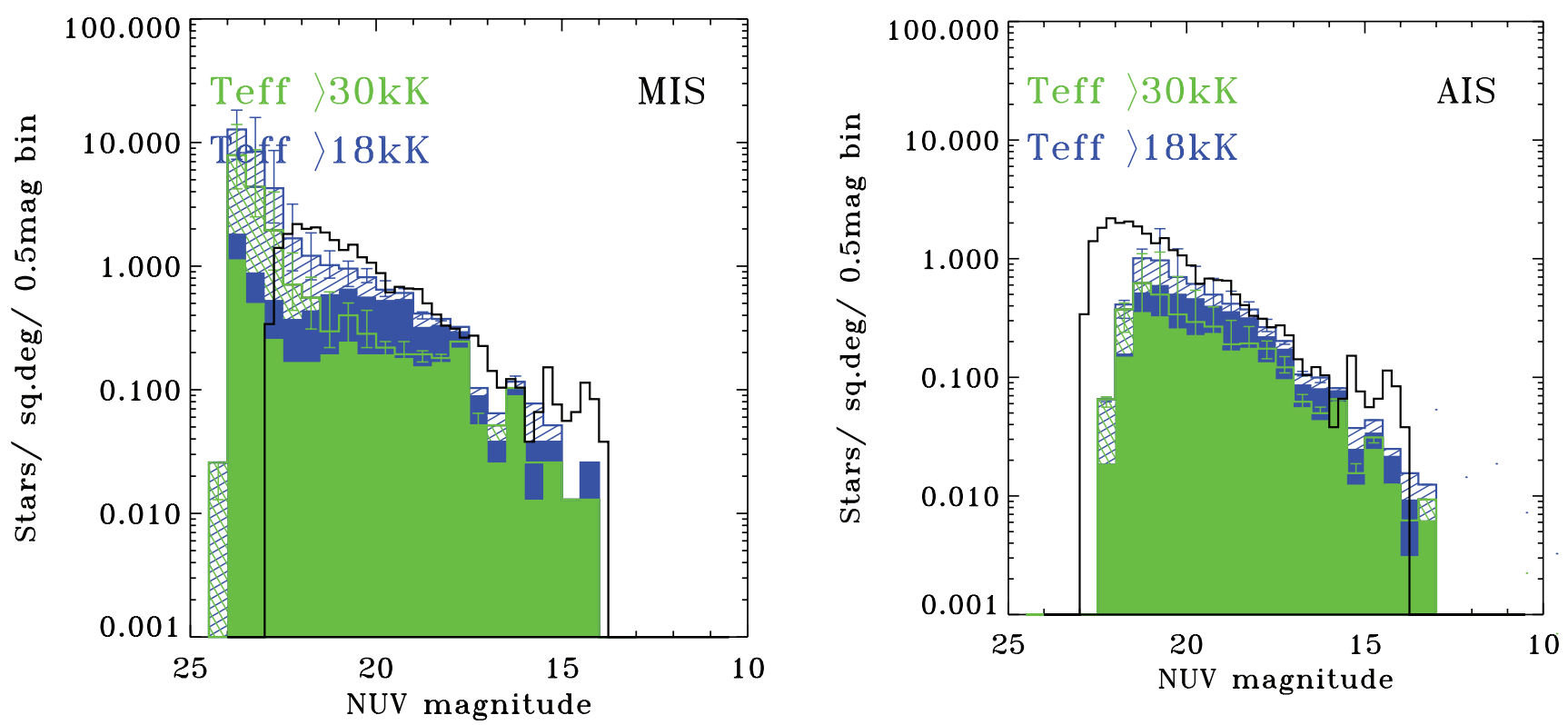

FIG. 10.-NUV-band surface density of the hot stars selected from FUV - NUV GALEX color, same sample as in the previous figure. Solid-color histograms show "single" hot WD candidates, dashed histograms include both single WDs and objects with optical colors redder than what corresponds to a hot WD with the observed UV color. These include binaries with a hot WD and possibly also extragalactic objects as suggested by their number increasing at fainter magnitudes. The Galaxy $r$-band WD model (black lines) has been shifted using an average color of $\langle\mathrm{NUV}-r\rangle=-1.5 \mathrm{mag}$. Because the UV band is more representative of the WD component in the case of binaries, the AIS and MIS counts at bright magnitudes are consistent as we expect, until the AIS becomes incomplete. The AIS is better sampled because it covers a larger area.

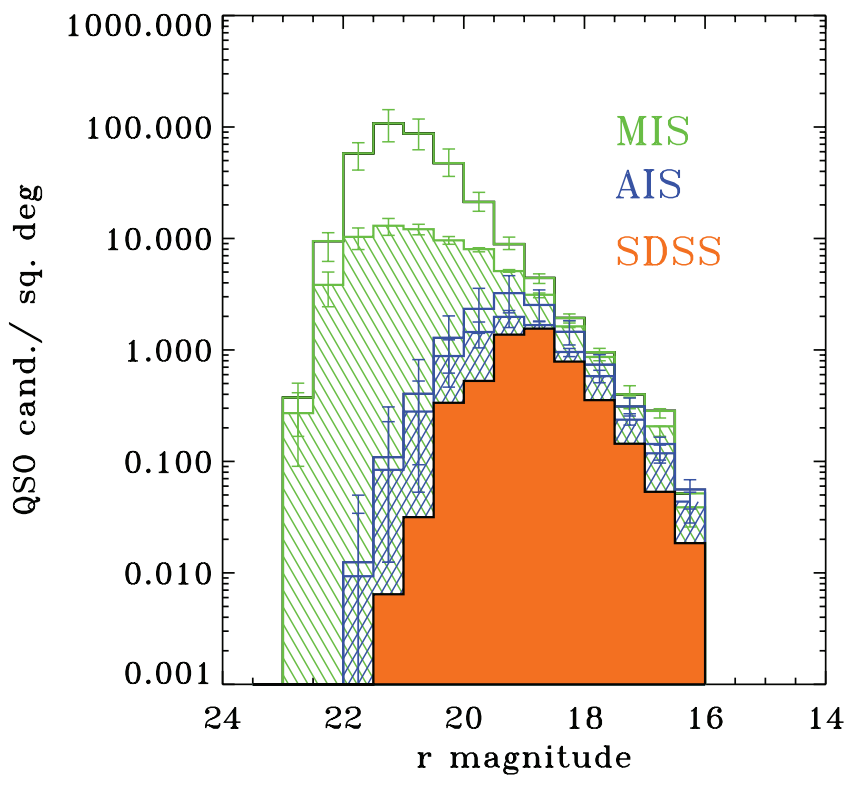

Fig. 11.- Surface density of low-redshift QSO candidates from the GALEX catalogs. In green, sources extracted from the deeper MIS; in blue, QSO candidates from AIS. Histograms only outlined include both pointlike and extended sources in the color locus of the QSOs. Shadow-filled histograms include only pointlike sources with the same color selection. Error bars are shown. The solid orange histogram shows the SDSS QSO catalog of Schneider et al. (2005); included are QSOs with $(z<1.3)$, to match the GALEX color selection. Including QSOs at all redshifts from the SDSS catalog does not change significantly the SDSS histogram on our scale. Note that the SDSS QSOs are spectroscopically confirmed; they are about $60 \%-85 \%$ of the SDSS QSOs candidates selected photometrically. We estimate that our photometric selection may contain $15 \%$ spurious sources. down to a radius of $\approx 0.05-0.02 R_{\odot}$, becoming fainter by -2.0 in $\log$ flux, or $5 \mathrm{mag}$.

Therefore, with error limits of $<0.15 / 0.3 \mathrm{mag}$ (FUV) and $<0.10 / 0.3 \mathrm{mag}$ (NUV) that translate into GALEX magnitude limits of $\approx 19.5 / 21.5 \mathrm{mag}$ for AIS and 22.5/25 mag for MIS, we expect that our GALEX data set can detect WDs all the way in the Galactic halo, even in the AIS sample, in their (advanced) postAGB constant luminosity phase. In the MIS sample, we expect to also detect hot WDs with radii as small as $0.04 R_{\odot}$ out to $20 \mathrm{kpc}$. When descending on the WD cooling sequence, the stars will have higher gravity. For $\log g=9.0$, FUV $-r=-2.33(-2.61)$ and NUV $-r=-1.73\left(-1.92 ; \mathrm{AB}\right.$ mag) for $T_{\text {eff }}=50,000$ and $100,000 \mathrm{~K}$, respectively, not significantly different from the $\log g=7$ case. Imposing an error limit of better than 0.1 mag in the $r$ and $g$ bands would, however, limit the survey to a smaller volume (see Fig. 3). These model magnitude values are in absence of reddening. However, as we saw from the color-color diagrams, reddening is quite small for the high Galactic latitudes of the present sample.

For subdwarfs and MS stars, e.g., with $\log g=5$ and $T_{\text {eff }}=$ 50,30 , and $18 \mathrm{kK}, \mathrm{FUV}-r=[-2.30,-1.87$, and -0.55$]$, $\mathrm{NUV}-r=[-1.74,-1.38$, and -0.40$]$. The radius will be larger than that of a WD, therefore all hot stars are expected to be detected in our GALEX surveys. For a reference radius of $1 R_{\odot}$, at $20 \mathrm{kpc}$ a star with $\log g=5.0$ and $T_{\text {eff }}=50,30$, and $18 \mathrm{kK}$ would have $r=16.53,17.34$, and $18.26 \mathrm{mag}$, respectively. These numbers are based on our TLUSTY model calculations. Using Kurucz models for $\log g=5$ stars, we find consistent magnitudes within $0.1 \mathrm{mag}$.

Here we use two different color selections to extract hot star candidates from the GALEX sources. First, the color-color diagrams NUV $-g$ versus $g-i$ or FUV $-g$ versus $g-i$ (Fig. 5), and the similar NUV $-g$ versus $g-r$ and FUV $-g$ versus $g-r$, can be used to select hot stars. At the hottest temperatures, MS and supergiant stars cannot be separated, but high-gravity stars (WDs) are well separated and are the majority of the hottest 
sources. At lower $T_{\text {eff }}$, MS stars and TO stars dominate the statistics (Fig. 5). Figure 8 shows the number per square degree of high-gravity stars, and lower gravity stars, selected from the NUV $-g$ versus $g-r$ colors, with error cuts of 0.1 mag (NUV, $r$, and $g$ ). If we included sources with larger errors, they would also be consistent with the locus of QSOs in the NUV $-g$ versus $g-r$ and FUV $-g$ versus $g-r$ diagrams. In this selection, hot stellar sources with a cool companion (occupying the top right part of the color-color diagrams) cannot be included, because for $g-r>-0.1$ they would be confused with QSO candidates. Therefore, our selection included the locus around the stellar sequence for high gravity (Fig. 5, purple symbols) down to $T_{\text {eff }} \approx$ $15,000 \mathrm{~K}$ for WDs. For the lower gravity stars, we included all the lower $T_{\text {eff }}$ sequence and no $g-r$ cut below $T_{\text {eff }}=10,000 \mathrm{~K}$, so binaries are also counted in this case.

The error bars on the number densities in Figure 8 are calculated by considering combined photometric $1 \sigma$ errors of each object, and counting the number of sources that fall inside and outside the color boundary that define an object class, when their individual errors are applied in both directions.

In Figure 8 we also plot predictions for the surface density of such objects calculated with the Besançon Milky Way model (Robin et al. 2003). We assumed a standard diffuse absorption of $A_{V}=0.7 \mathrm{kpc}^{-1}$ and calculated the predictions over an area of approximately $210 \mathrm{deg}^{2}$ between $40^{\circ}$ and $50^{\circ}$ in Galactic latitude. No population age selection has been applied. The calculations were extended to $g=24$. The Milky Way model predictions are shown as black histograms, with symbols of the same color of the objects they represent (green for WD, blue for MS and TO, and orange for total) in the data histograms. The lower than predicted number of bright objects is due to saturation in the SDSS bands (saturated objects are excluded in our sample), which occur around 14th mag (between 13 and 15 mag). At the fainter magnitude end, incompleteness is due to the error cuts.

We also performed a selection of hot WD candidates based on the FUV - NUV color only, down to $T_{\text {eff }} \approx 18,000 \mathrm{~K}$. Below this limit their FUV - NUV color overlaps with the $z \approx 0$ QSOs; our QSO templates with $z=0$ have FUV $-\mathrm{NUV}=0.175 \mathrm{mag}$.

We used [FUV $-\mathrm{NUV}]<-0.037 \mathrm{mag}$ (corresponding to $T_{\text {eff }}>18,000 \mathrm{~K}$ for $\log g=5$ ) and FUV - NUV $<-0.343$ mag, which corresponds to $T_{\text {eff }}>30,000 \mathrm{~K}$ for $\log g=5$ but includes objects down to $T_{\text {eff }} \approx 25,000 \mathrm{~K}$ for higher gravities. The surface density of the hot stars selected in this way, from GALEX measurements only (errors $<0.3 \mathrm{mag}$ ), is shown in Figures 9 and 10. If we restrict the sample to sources with $\operatorname{err}_{\mathrm{FUV}}<0.15 \mathrm{mag}$ and $\operatorname{err}_{\mathrm{FUV}}<0.10 \mathrm{mag}$, the shape of the histogram does not change significantly, but the density of objects becomes about half in both AIS and MIS, and the counts begin to drop at about 1 mag brighter than they do in Figure 9.

The WD surface density in Figure 9 matches the predictions by the Milky Way model in the MIS sample and is a factor of 2 lower in the AIS sample. However, we suspect that the MIS counts are contaminated by pointlike sources possibly of extragalactic nature, as explained and quantified below. Such spurious sources affect only the "binary" candidates, and not the "single WD" candidates, as we see below.

The number of objects per square degree (surface density) are plotted in Figures 8 and 9 as a function of the visual $r$ magnitude, for direct comparison with the Galactic model predictions and with other works. When we combine the GALEX selection of hot stellar objects (from FUV - NUV color) with optical bands, we can also assess the fraction of hot stars that have a cooler companion, i.e., objects with FUV - NUV color corresponding to a hot $T_{\text {eff }}$, but much redder optical colors. However, objects with
WD-like FUV - NUV colors and brighter than expected optical magnitudes may also be of extragalactic origin, such as unresolved galaxies or QSOs whose SEDs may differ from our templates. The fraction of objects with optical colors inconsistent with their hot-WD FUV - NUV color, is about $20 \%-30 \%$ for magnitudes brighter than $\approx 20$, however the number substantially increases at fainter magnitudes. This increase suggests some contamination by nonstellar (extragalactic) objects. The $r$ magnitude of a fraction of our sample objects therefore does not correspond to that of the WD component, but rather to the optical magnitude of a cool companion, or to that of a different object in case of extragalactic contaminants. In either case, the observed $r$ magnitude is brighter than the WD optical magnitude. This fact causes an artificial increase in the number of MIS sources in the bright $r$ magnitudes range, where we would expect that the density of sources (either single WD, binaries, or possible spurious objects) would anyway be the same in AIS and MIS, down to the limit where the AIS begins to be incomplete. Therefore, we consider the match of the Milky Way model to the MIS counts in Figure 9 (left) to be, at least in part, a bias from this effect. In order to avoid this bias, we plotted in Figure 10 the surface density for the same sample of hot-star candidates as a function of NUV magnitude, which better represents the magnitude of the WD component in the binary objects. The solid-color histograms show the "single" objects (whose optical colors are consistent with the FUV - NUV hot-star classification). These histograms show similar object counts for MIS and AIS, down to where the AIS becomes incomplete, as one would expect. The dashed histograms show all FUV - NUV selected sources, both single- and binary-WD candidates, the latter including possible spurious objects.

These total counts increase at fainter magnitudes much more than the single-WD counts, reinforcing the suspicion of contamination by extragalactic objects in the "binaries" sample. Because the Besançon Milky Way model is not available specifically for GALEX bands, we have taken the model prediction in the $r$ band and translated it into the NUV band using an average NUV $-r$ color in the range of $T_{\text {eff }}$ where our WD candidates were selected, $\langle\mathrm{NUV}-r\rangle=-1.5 \mathrm{mag}$. Such approximation only provides a qualitative comparison, however we believe it is quite close to reality since the Galaxy model includes WDs in binaries in the predicted counts but does not account for the color effects of the companion stars.

The good qualitative match between the observed surface density of objects and the Milky Way model, confirms our prediction (see above) that GALEX can detect hot WDs throughout the Galactic halo. The GALEX selection of high-gravity hot stars in the Galaxy offers therefore a major improvement with respect to previous surveys.

For comparison to previous catalogs based on extensive surveys, we recall that the Acker et al. (1982) catalog of Galactic planetary nebulae includes 36 (12) objects at latitudes higher than $30^{\circ}\left(45^{\circ}\right)$, out of a total of $\approx 1140$ objects. McCook \& Sion (1999) catalog of WDs has 2364 (1553) WDs at latitudes higher than $30^{\circ}\left(45^{\circ}\right)$, out of $\approx 3060$ total objects. Kleinman et al. (2004) catalog 2551 certain white dwarf stars, 240 hot subdwarf stars, and another 144 possible, but uncertain, white dwarf and hot subdwarf stars from the $1360 \mathrm{deg}^{2}$ of SDSS DR 1, or about 2.2 objects per square degree. The Palomar-Green catalog of UVexcess stellar objects (Green et al. 1986), covering 10,714 $\mathrm{deg}^{2}$, lists 1874 objects with limiting magnitudes between $B=15.49$ and 16.67. Eisenstein et al. (2006) catalog about 9316 WDs and 928 subdwarfs from the SDSS Data Release 4, covering $4783 \mathrm{deg}^{2}$. The GALEX selection of hot stellar objects is more sensitive to the hottest objects, and especially to binaries containing a hot 
WD, since the optical photometry in such cases is dominated by the cooler companion (see also Bianchi et al. 2006, Fig. 6, for an example).

The question of contamination of the hot-star candidate sample by nonstellar pointlike sources can be better investigated with follow-up spectroscopy, which is under way. In order to assess the robustness of our photometric selection, we have matched our samples of photometrically selected hot stars with the SDSS Data Release 5 (DR5) spectroscopic data.

The spectroscopic survey is less deep than the photometric survey, so the fraction of our hot-star-selected samples that have available spectra in DR5 is higher for the AIS samples than for the MIS samples. Of the hot stars $\left(T_{\text {eff }}>18,000 \mathrm{~K}\right)$ selected from the FUV - NUV color (Fig. 10), 28\% (AIS), and 5\% (MIS) have optical spectra. In particular, of the "single" hot-star candidates (whose optical colors are consistent with the UV colors, and are plotted as solid-color histograms in Fig. 10), 29\% (AIS) and 16\% (MIS) have SDSS spectra. Of our sample "binaries" (which may include both true stellar binaries with a WD plus cool companion and spurious objects), 24\% (AIS) and 1\% (MIS) have spectra. Therefore, the statistics are more significant for AIS and for "single" WD selections than for other samples. Of the sources with spectroscopic classification, $97 \%$ (AIS) $/ 95 \%$ (MIS) of our "single" hot-star candidates are confirmed as stars. Out of the "binary" candidate sample, $45 \%$ (AIS) and 31\% (MIS) are spectroscopically classified as stars, $47 \%$ (AIS) and 65\% (MIS) are spectroscopically classified as QSOs. The fractions are very similar for the sample of hot stars with $T_{\text {eff }}>30,000 \mathrm{~K}$. These numbers confirm our previous conclusions, based on the photometrically histograms of density counts, that FUV - NUV selected hot stars with inconsistent optical colors include both true stellar binaries and extragalactic objects with SED differing from the average QSO templates shown in our color-color diagrams. More importantly, they indicate that our FUV - NUV selection of "single" hot stars is quite robust.

As for the hot stars (WDs) selected from the NUV $-g$ versus $g-r$ diagram, and shown in the histograms of Figure 8, $57 \%$ (AIS) $/ 40 \%$ (MIS) have SDSS spectra. Of these, $66 \%$ (AIS)/ $61 \%$ (MIS) are spectroscopically confirmed as stars; $30 \%$ (AIS)/ 34\% (MIS) are spectroscopically classified as QSOs. We believe that the purity of the stellar sample is lower in this case because the locus used to extract the high-gravity stars includes lower $T_{\text {eff }}$ values than our FUV - NUV selection, and it is close to the QSO locus.

\subsection{QSO Candidates}

As pointed out previously by Bianchi et al. (2005a), the FUV NUV versus NUV - $r$ diagram (Fig. 7) can also be used to select low-redshift QSOs candidates. Figure 11 shows the observed surface density of low-redshift QSO candidates, selected from this color combination from both the MIS and AIS surveys. For comparison, the recent QSO catalog of Schneider et al. (2005) from the SDSS DR3-release is also shown (orange histogram). To normalize the SDSS QSO catalog to density of objects per unit area, we used an area of $3732 \mathrm{deg}^{2}$, the coverage of the spectroscopic DR3 release, because the catalog includes spectroscopically confirmed QSOs. We also limited the SDSS catalog to QSOs with $z<1.3$, corresponding to our GALEX photometric selection. To actually scale the SDSS spectroscopically confirmed catalog to the SDSS photometric selection of QSO candidates, we must consider that about $60 \%$ of the photometrically selected candidates have been observed spectroscopically so far (G. Richards 2005 , private communication), although the exact fraction is not available from the Schneider et al. (2005) catalog. For more discussion, see Richards et al. (2005) and Hutchings et al. (2008, in preparation). The density of objects in our GALEX photometrically selected sample is larger than the SDSS QSO candidate sample and extends to fainter magnitudes.

In Figure 11, we show separately the pointlike QSO-candidate GALEX sources, and all sources with QSO-like colors regardless of spatial extent, i.e., including extended sources that have observed colors consistent with our photometric selection. The present classification of "pointlike" and "extended" is based on the SDSS $\approx 1.4^{\prime \prime}$ PSF and obtained from the pipeline.

However, the contrast between the AGN central source and the underlying galaxy is still poorly characterized at low redshifts $(z \approx 1)$. The GALEX-selected catalog of low-redshift QSO candidates presents an opportunity to clarify this issue, which will be investigated with follow-up deeper imaging.

We finally point out that other objects share the locus of the QSOs in the color-color diagram: cataclysmic variables (CVs) with a significant accretion disk have similar colors and thus potentially contaminate the QSO sample. These objects, however, are extremely rare, about $0.02 \mathrm{deg}^{-2}$ (e.g., Szkody et al. 2004 and references therein). Our QSO candidate density is at least 1 order of magnitude higher than the expected density of CVs in the AIS and about 2 dex higher in the MIS; therefore, we expect our $G A L E X$-selected QSO candidates to not be significantly contaminated. However, such stellar sources (expected to be nearby and therefore bright) might explain the excess of sources at bright magnitudes, with respect to the confirmed SDSS QSOs. To estimate the purity of the photometrically selected sample, as we did for the hot star samples, we have matched our QSO candidates with the SDSS DR5 to search for archival optical spectra. We found that $60 \%$ (AIS) $/ 17 \%$ (MIS) of our QSO candidates (pointlike) have spectra, and of these, $83 \%$ (AIS) $/ 85 \%$ (MIS) confirm the QSO classification. The statistics is fairly significant: $1631 \mathrm{ob}-$ jects have spectra out of 2689 total objects for AIS, 930 out of 5312 for MIS. Of the non-QSO objects, $14 \%$ (AIS)/12\% (MIS) are spectroscopically classified as stars, consistent with what one can qualitatively expect from the color-color diagrams. Therefore, based on the results from the SDSS automated spectral classification, the purity of our GALEX-selected pointlike QSOcandidate sample can be considered $\approx 85 \%$, comparable or higher to the success rate of optical selection. As for the extended sources that fall in our QSO-selection color locus, only $19 \%$ (AIS) $/<0.1 \%$ (MIS) have spectra: of these, $27 \%$ (AIS) $/ 48 \%$ (MIS) are spectroscopically classified as QSOs. The statistic is less significant than for the pointlike sources.

GALEX (Galaxy Evolution Explorer) is a NASA Small Explorer, launched in 2003 April. We gratefully acknowledge NASA's support for construction, operation, and science analysis of the GALEX mission, developed in cooperation with the Centre National d'Etudes Spatiales of France and the Korean Ministry of Science and Technology. We are grateful to John Hutchings, Wei Zheng, and Gordon Richards for discussions about QSO issues and clarifications about the QSO SDSS catalogs and templates, to Alessandro Bressan for providing the yet unpublished SSP models and for extremely useful discussions, and (with Olga Vega) for assistance in calculations of the galaxy template, to Paula Szkody and Knox Long for illuminating discussions about CVs and for the CV templates.

Facilities: GALEX, Sloan 
Abazajian, K., et al. 2005, AJ, 129, 1755

Acker, A., Gleizes, F., Chopinet, M., Marcout, J., Ochsenbein, F., \& Roques, J. M.

1982, Publ. Spéc. Cent. Données Stellaires, 3, 4

Bianchi, L., \& Efremova, B. 2006, AJ, 132, 378

Bianchi, L., \& Garcia, M. 2002, ApJ, 581, 610

Bianchi, L., et al. 2005a, ApJ, 619, L27 2005b, BAAS, 37, 1385

2006, in The UV Universe: Stars from Birth to Death, ed. A. I. Gomez de Castro \& M. Barstow (Madrid: Univ. Complutense de Madrid), in press Bressan, A., Granato, G. L., \& Silva, L. 1998, A\&A, 332, 135

Budavari, T., Conti, A., Seibert, M., Szalay, A. S., \& GALEX Science Team. 2004, BAAS, 36, 1410

de Vaucouleurs, G., de Vaucouleurs, A., Corwin, H. G., Jr., Buta, R. J., Paturel, G., \& Fouque, P. 1991, Third Reference Catalogue of Bright Galaxies (Berlin: Springer)

Eisenstein, D. J., et al. 2006, ApJS, 167, 40

Francis, P. J., Hewett, P. C., Foltz, C. B., Chaffee, F. H., Weymann, R. J., \& Morris, S. L. 1991, ApJ, 373, 465

Fukugita, M., Ichikawa, T., Gunn, J. E., Doi, M., Shimasaku, K., \& Schneider, D. P. 1996, AJ, 111, 1748

Garcia, M., \& Bianchi, L. 2004, ApJ, 606, 497

Green, R. F., Schmidt, M., \& Liebert, J. 1986, ApJS, 61, 305

Hubeny, I., \& Lanz, T. 1995, ApJ, 439, 875
REFERENCES

Kleinman, S. J., et al. 2004, ApJ, 607, 426

Lejeune, T., Cuisinier, F., \& Buser, R. 1997, A\&AS, 125, 229

Martin, D. C., et al. 2005, ApJ, 619, L1

McCook, G., \& Sion, E. 1999, ApJS, 121, 1

Morrissey, P., et al. 2005, ApJ, 619, L7

- 2007, ApJS, 173, 682

Oke, J. B., \& Gunn, J. E. 1983, ApJ, 266, 713

Pauldrach, A., Hoffman, T. L., \& Lennon, M. 2001, A\&A, 375, 161

Pier, J. R., Munn, J. A., Hindsley, R. B., Hennessy, G. S., Kent, S. M., Lupton, R. H., \& Ivezić, Ž. 2003, AJ, 125, 1559

Pourbaix, D., et al. 2004, A\&A, 423, 755

Raymond, S. N., et al. 2003, AJ, 125, 2621

Richards, G. T., et al. 2005, MNRAS, 360, 839

Robin, A. C., Reylé, C., Derrière, S., \& Picaud, S. 2003, A\&A, 409, 523 (erratum 416, 157 [2005])

Rodriguez-Merino, L. H., Chavez, M., Bertone, E., \& Buzzoni, A. 2005, ApJ, 626, 411

Schneider, D. P., et al. 2005, AJ, 130, 367

Silva, L., Granato, G. L., Bressan, A., \& Danese, L. 1998, ApJ, 509, 103

Smolčić, V., et al. 2004, ApJ, 615, L141

Szkody, P., et al. 2004, AJ, 128, 1882

Yi, S. K. 2003, ApJ, 582, 202

York, D. G., et al. 2000, AJ, 120, 1579 